\title{
Zooplankton trajectory before, during and after a hydropower dam construction
}

Trajetória do zooplâncton antes, durante e depois da construção de uma barragem de hidroelétrica

Jaqueline Schmidt ${ }^{1}$ (D), Patrícia Dammski Borges de Andrade ${ }^{2}$ (D) and André Andrian Padial ${ }^{1 *}$

${ }^{1}$ Laboratório de Análise e Síntese em Biodiversidade, Departamento de Botânica, Setor de Ciências Biológicas, Universidade Federal do Paraná - UFPR, Av. Cel Francisco Heráclito dos Santos, s/n, Jardim das Américas, CEP 81531-990, Curitiba, PR, Brasil

${ }^{2}$ LACTEC Instituto de Tecnologia para o Desenvolvimento, Rodovia BR-116, Km 98, 8813, Prédio CEHPAR, Jardim das Américas, CEP 81531-980, Curitiba, PR, Brasil

*e-mail: aapadial@gmail.com

Cite as: Schmidt, J., Andrade, P.D.B. and Padial, A.A. Zooplankton trajectory before, during and after a hydropower dam construction. Acta Limnologica Brasiliensia, 2020, vol. 32, e18.

Abstract: Aim: Understanding the impact of anthropogenic activities is central for supporting management and conservation efforts. In aquatic ecosystems, the construction of dams for hydroelectric power plants is a major environmental change that turns the riverine ecosystem into a reservoir lake. Such environmental deep alteration causes profound impacts in biota. The goal of this study is to make a comprehensive description of zooplankton trajectory following the construction of a reservoir in the transition from the hotspot Cerrado to Amazon, Central Brazil. Methods: We used data sampled before, during and after the formation of the reservoir lake in 10 sampling units each period. We evaluated compositional changes, shifts in spatial organization, and a variation in beta-diversity from before to after the dam constructions using a set of multivariate analyses. We evaluated effects for Rotifers, Copepods and Cladocerans separately. Results: Compositional changes were evident for all zooplankton groups: Rotifers, Copepods and Cladocerans. Besides, spatial community organization was also affected but depending on the beta-diversity facet and data resolution - mainly turnover using abundance data, except for Copepods. Finally, an increase in nestedness occurred for all groups during the formation of the reservoir lake. Conclusions: In summary, our study showed the deep impacts for zooplankton that the formation of a reservoir lake causes. We innovate by making a complete assessment, which indicate clearly the complexity of evidencing impacts in aquatic communities. We also suggest that long-term monitoring should continue in reservoirs for scientific purposes. The changes in biota also make clear that the construction of dams should be accompanied by preservation of other pristine riverine ecosystems.

Keywords: metacommunity; beta-diversity; biotic homogenization; microcrustaceans; reservoirs; Anthropocene.

Resumo: Objetivo: $\mathrm{O}$ entendimento do impacto de atividades antrópicas é essencial para subsidiar esforços de manejo e conservação. Em ecossistemas aquáticos, a construção de barragens para usinas hidroelétricas é uma das principais alterações ambientais que tornam o ecossistema fluvial em um reservatório. Tal alteração ambiental causa uma alteração profunda na biota. $\mathrm{O}$ objetivo desse estudo é fazer uma descriçấo completa da trajetória da comunidade zooplanctônica devido à construção de um reservatório na transição entre o hostpot brasileiro Cerrado e a Amazônia, no Brasil Central. Métodos: Foram utilizados dados amostrados antes durante e depois da formação do lago 
do reservatório coletados em 10 pontos em cada um desses períodos. Foram avaliadas as mudanças composicionais, as alteraçóes na organização especial e as variaçóes da diversidade-beta entre antes e depois da construçáo do reservatório usando um conjunto de análises multivariadas. Efeitos em Rotíferos, Copépodos e Cladóceros foram avaliados separadamente. Resultados: Mudanças na composição de espécies foram evidentes para todos os grupos zooplanctônicos: Rotíferos, Copépodos e Cladóceros. Além disso, a organização espacial das comunidades também foi afetada, dependendo da faceta de diversidade beta e da resolução numérica dos dados - principalmente turnover usando dados de abundância, exceto para Copépodos. Finalmente, houve aumento de aninhamento da comunidade durante o enchimento do reservatório para todos os grupos zooplanctônicos. Conclusóes: Em resumo, nosso estudo mostrou os impactos que a formação do lago de reservatórios causa na comunidade zooplanctônica. O estudo é inovador por fazer uma descrição completa, que claramente indica a complexidade em evidenciar impactos nas comunidades ecológicas aquáticas. Também se sugere que o monitoramento de longo prazo deve continuar em reservatórios para fins científicos. As mudanças na biota deixam claro que a construção de barragens deve ser acompanhada pela preservação de outros ecossistemas fluviais pristinos.

Palavras-chave: metacomunidade; diversidade-beta; homogeneização biótica; microcrustáceos; reservatórios; Antropoceno.

\section{Introduction}

Human society is highly dependent in electric power, which generation represents a major source of environmental impacts. Compared to nations that generate power mainly by fossil fuels, which has strong environmental impacts associated to atmospheric pollution and greenhouse gases, Brazil has a relatively sustainable matrix of electric power generation - based mainly in hydropower dams (Von Sperling, 2012). Even so, such generation do represent impact for natural ecosystems, given the strong environmental changes that dam construction represents both considering socio-economic features to communities living nearby water bodies (Von Sperling, 2012), as well as to aquatic ecosystem functioning, habitat fragmentation and freshwater biodiversity (Baxter, 1985; Agostinho et al., 2008).

Any human intervention in natural ecosystems cause ecological impacts, but the extent of impacts, the sustainability of interventions and the information to society still need to be improved for better governmental decisions (Azevedo-Santos et al., 2017). Therefore, ecological studies monitoring biodiversity changes are central. Not surprisingly, understanding causes and patterns of spatial and temporal variation in ecological communities is a major goal of Community Ecology that informs ecosystem conservation (Socolar et al., 2016). When describing impacts of dam constructions, before and after sampling design is a common approach (Agostinho et al., 2008; Vieira et al., 2019). Indeed, although causal inference and impact evaluation in ecology is difficult, before and after sampling design is the most suitable to infer anthropogenic impacts, such as reservoir damming. The most complete approach, however, would be to compare before and after trajectories in impacted ecosystems with trajectories in comparable non-impacted ecosystems (see Ribas et al., 2019)

Immediate effects of dams in several ecological communities have been described (e.g. Agostinho et al., 2008; Pelicice et al., 2015; Silva et al., 2017; Vincentin et al., 2018; Noleto et al., 2019). Although zooplankton is a key biological group that responds quickly to environmental alterations and very important to ecosystem functioning, this groups is relatively less studied compared to fish, together with macroinvertebrates (Figure 1). In a metacommunity perspective, one can expect that environmental alterations may affect distinct groups of zooplankton differently (Soares et al., 2015). For instance, due to small size, quick response to environmental conditions and due to being more sessile (see also Soares et al., 2015), Rotifers can probably be more affected than micro-crustaceans (Cladocerans and Copepods) considering composition changes before and after dam constructions. Moreover, impact may not only be reflected by compositional changes, but also in changes in compositional variation another facet of beta diversity (i.e. mission statements 2 vs. mission statement 4 in Anderson et al., 2011). As described in Anderson et al. (2011), a common goal is to explore relationships between community structure and environmental factors: evaluating compositional changes before and after a dam construction would represent this goal. Another goal in a higher level of abstraction would be comparing variation in community structure among groups: for instance, compositional variation over space among the periods before and after. 
The environmental homogenization caused by the formation of the reservoir lake may also reflect in a higher spatial similarity of mainly micro-crustaceans communities. If this is the case, the natural spatial variation that occur along a river system, in response to environmental heterogeneity and migration can be disrupted, and the formation of the reservoir lake could be compared to the flood-homogenization that occurs in floodplain systems (see an example for zooplankton in Bozelli et al., 2015). Indeed, micro-crustaceans (particularly Copepods) seem to be relatively more related to environmental features (Zhao et al., 2017). If so, one can expect changes in spatial organization (not community composition) and possible biotic homogenization phenomenon after the anthropogenic impact (e.g. Olden \& Rooney, 2006).

We evaluated the temporal changes in zooplankton communities in a reservoir installed at a river from a major tributary of Amazon Basin. Given the amount of hydropower dams proposed in Amazon (Winemiller et al., 2016), our study is timely to describe effects of river damming in a central regions for biodiversity conservation in Brazil. We analyzed different facets of zooplankton biodiversity that may better inform conservation and ecological monitoring (Socolar et al., 2016).

\section{Methods}

\subsection{Study site}

Data used in this study was sampled in the 'Programa de Monitoramento da Qualidade da Água de reservatórios da COPEL' carried out by the Research and Technology Institute called 'Lactec - Instituto de Tecnologia Para o
Desenvolvimento'. Sampling occurred before, during and after the construction of the hydropower known as 'UHE Colíder', under responsibility of the company from the 'Companhia Paranaense de Energia - COPEL'. The hydropower dam is located in 'Mato Grosso State' at the transition zone between the hotspot Cerrado and Amazon (Mid-West Brazil), at 'Nova Canaã do Norte' and 'Itaúba' municipalities, but also affecting areas from other two municipalities (COPEL, 2018). The impacted 'Telles Pires' River is one of the rivers that generate the 'Tapajós' River, which in turn is a major tributary of the Amazon River (see Figure 2 for a detailed location). The electric generation power of UHE Colíder is $300 \mathrm{Mega-Watts} \mathrm{(enough}$ for a city with 850 thousand people, Wosiack et al., 2018); and the reservoir encompasses an area of $171.7 \mathrm{~km}^{2}$. The length of the reservoirs is $94 \mathrm{~km}$ from the dam to the beginning of the lake. Although the reservoir is not among the largest in Amazon basin, it is one of a series of four medium-sized reservoirs that together may represent a large impact in a major tributary of Amazon Basin. Indeed, although large sized reservoirs represent major 'per capita' impact, the numerous and widespread small and medium reservoirs may represent a great impact in most basins (Couto \& Olden, 2018).

\subsection{Samplings}

Samplings before the formation of the lake were carried out in 2016 (BF); during the formation of the lake in 2017 (DU); and after the formation in 2018 (AF). In each of sampling campaigns, 10 sampling units were monitored (see Figure 2, see that location of two sampling units were changed from the campaigns BF to the others; it was not
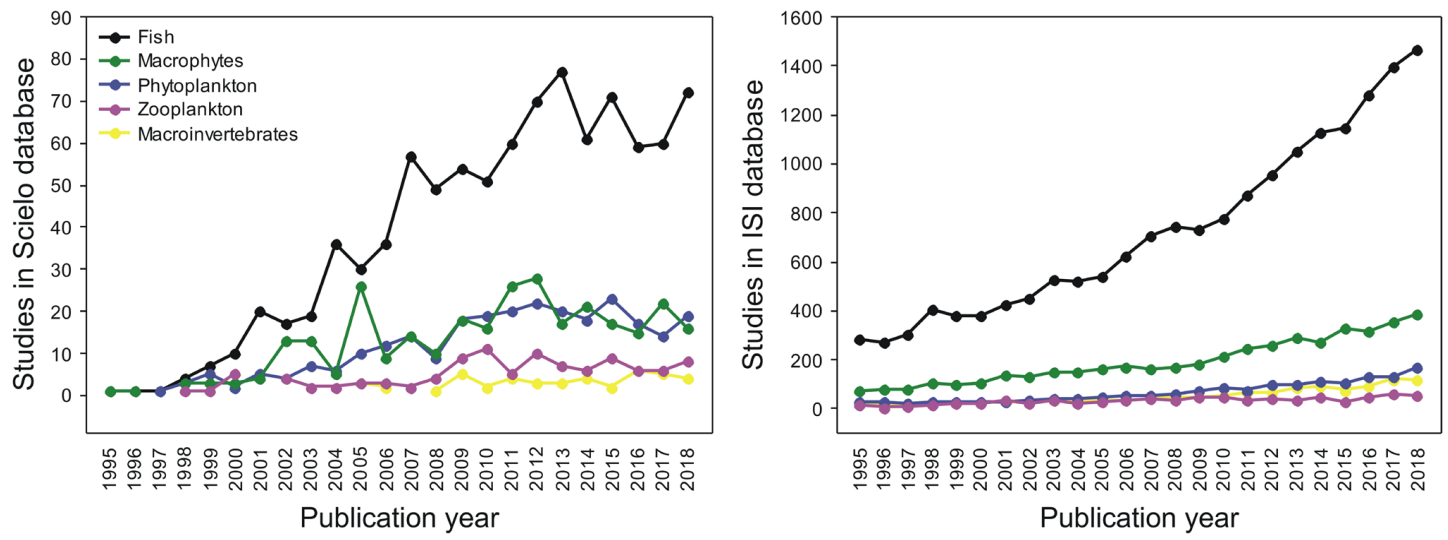

Figure 1. Number of papers returned in a search in Scielo and ISI (Web of Science) databases using the following words in topic: (dam* OR reservoir*) AND (fish* OR (macrophyte* OR 'aquatic plant*') OR (phytoplankt* OR algae) OR zooplankt* OR (macroinvert* OR zoobent* OR 'aquatic insect*')). 


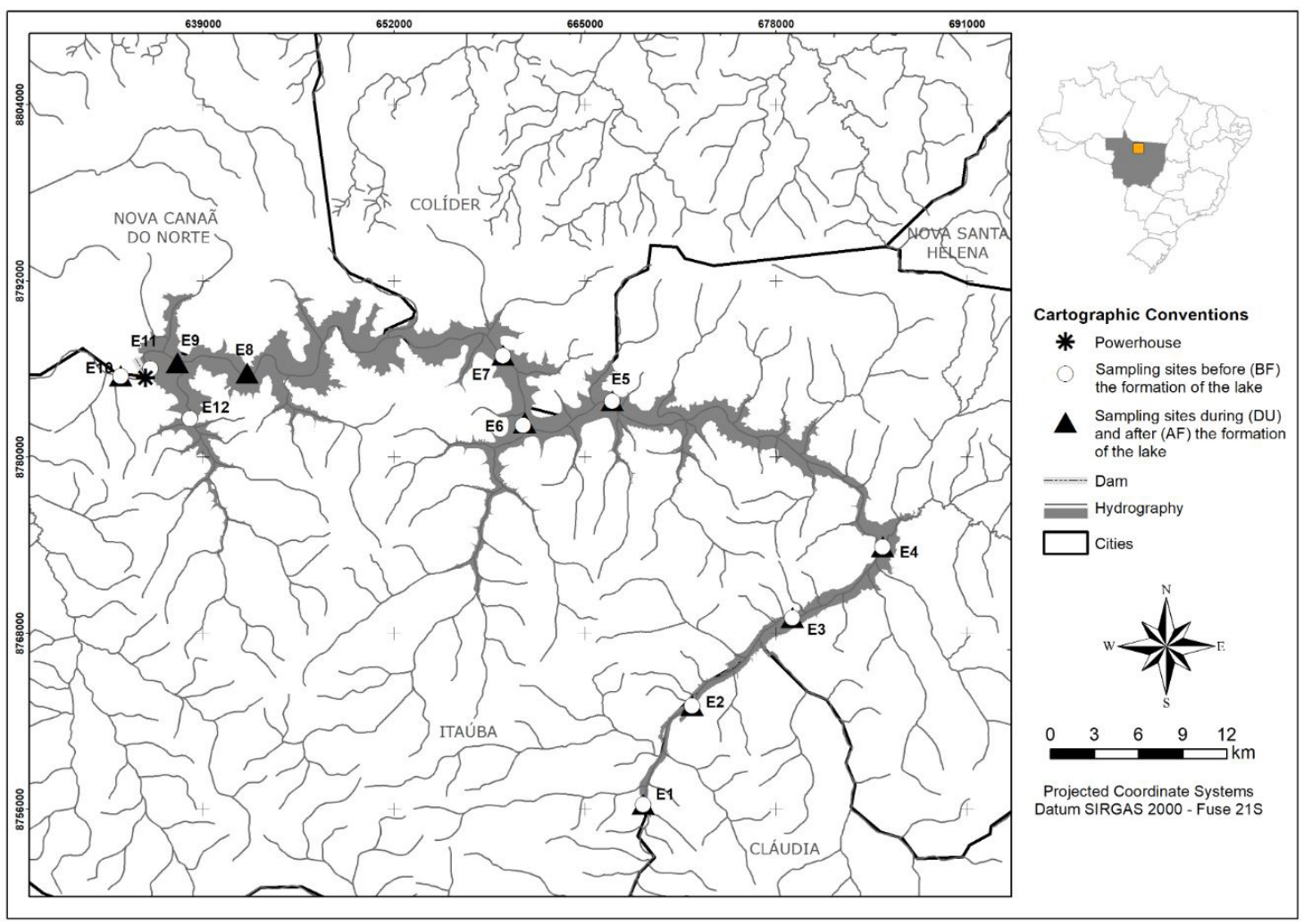

Figure 2. Map showing location of the hydropower dam and the sampling units. Note that two sampling units were modified considering sampling before to sampling during and after. Modified from Wosiack et al. (2018). E1 to E12 indicate location of the 12 sampling sites.

possible to sample at a same location given the landscape change). In each sampling unit, $400 \mathrm{~L}$ (BF) and $800 \mathrm{~L}$ (DU and AF) were filtered in a mesh of $64 \mu \mathrm{m}$. The volume differed to ensure that a similar number of individuals could be recorded in each campaign. Therefore, records were standardized by considering the filtered volume. Sampling material were stored in $500 \mathrm{~mL}$ plastic tubes and preserved in 95 Alcohol. At the lab, samplings were filtered again (mesh of $64 \mu \mathrm{m}$ ), and if necessary diluted to facilitate identification. Rose Bengal dye was used as an organism-coloring agent. A $1 \mathrm{~mL}$ concentrated sampled was then used for identification in Sedgwick-Rafter counting cell, and identification occurred in optical microscope using up to 100x objective lenses. Identification followed specialized literature (e.g. Ruttner-Kolisko, 1974; Silva et al., 1989; Shiel, 1995; Elmoor-Loureiro, 1997; Witty, 2004; Joko, 2011; Gazulha, 2012).

\subsection{Data analysis}

All analyses were carried out in $\mathrm{R}$ environment (R Core Team, 2017) using the packages 'vegan' (Oksanen et al., 2017), 'labdsv' (Roberts, 2016) and 'betapart' (Baselga et al., 2018). Hypothesis tests were considered significant if type I error were lower than 5\%. When multiple tests were done, type I error probability was divided by the number of tests used (popularly known as Bonferroni correction).

Changes in species compositions were analyzed using a PERMANOVA (Anderson, 2001) with 999 permutations. If significant, differences in BF, DU and AF were visualized in a Principal Coordinate Analysis (PCoA; Gower, 1966) and species typical from each period were identified using the Indicator Value Index (IndVal; Dufrêne \& Legendre, 1997). IndVal is an index that balances the fidelity (in our case, the occupation of the species in all samplings of a period) and specificity (in our case, the occurrence of the species in only one period) of each species in each classification (in our case, periods). IndVal varies from 0 (no fidelity and specificity) to 1 (maximum specificity and fidelity) (Dufrêne \& Legendre, 1997). We then compared IndVal for each species with a null expectation after 999 permutations. The spatial organizations of different periods (described by compositional dissimilarity matrices) were compared among periods using Mantel tests (Mantel, 1967) also with 999 permutations. A significant Mantel 
correlation would mean that spatial community organization was not disrupted among periods, contrarily to what we expect. Finally, variation in compositional dissimilarity (mission statement 4 in Anderson et al., 2011) was also evaluated by applying the betadipser with permutest approach applied in a PCoA (Anderson et al., 2006), also with 999 permutations. This approach estimates a value of variation in compositional dissimilarity calculated by the mean distance of each local community to the centroid of a metacommunity. Thus, we used this approach to estimate the total variation in metacommunity beta diversity for each period (BF, DU and AF).

All analyses were carried out using Hellinger-transformed (Legendre \& Gallagher, 2001) abundance and occurrence (i.e. presence/absence) data (except for IndVal, which uses only abundance to calculate the fidelity, see Dufrêne \& Legendre,
1997). As a consequence, Bray-Curtis and Sorensen dissimilarities were used, respectively, to generate dissimilarity matrices. In Mantel tests and betadisper approach, we compared spatial organization and community variation (respectively) using turnover and nestedness components (considering both abundance and occurrence) of beta diversity following Baselga (2010). This could show if impact affect a component of beta diversity different from another. All analyses were done separating Rotifers, Copepods and Cladocerans.

\section{Results}

164 taxa were identified in all samplings. The most common were Rotifers (102 taxa), followed by Cladocerans (41 taxa) and Copepods (21 taxa). A full table of taxa sampled in each period (BF, DU and $\mathrm{AF}$ ) is available as supporting information (Table 1).

Table 1. Full list of zooplankton taxa (separated by Rotifers, Copepods and Cladocerans) sampled before (BF), during (DU) and after (AF) the formation of the reservoir lake from the hydropower dam UHE Colíder.

\begin{tabular}{|c|c|c|c|c|c|c|c|}
\hline Rotifers & BF & DU & AF & Rotifers (...continuing...) & BF & DU & AF \\
\hline Ascomorpha sp. & $x$ & $x$ & $x$ & Pleosoma sp. & $\mathrm{X}$ & & \\
\hline Asplanchna brightwellii & & & $x$ & Ploesoma truncatum & & & $\mathrm{X}$ \\
\hline Asplanchna sieboldii & $\mathrm{X}$ & & & Polyarthra dolichoptera & $\mathrm{X}$ & & \\
\hline Asplanchna sp. & $x$ & $x$ & $x$ & Polyarthra remata & $x$ & & \\
\hline Bdelloidea & $x$ & $x$ & $x$ & Polyarthra sp. & $x$ & $x$ & $\mathrm{X}$ \\
\hline Beauchampiella sp. & $x$ & & $x$ & Polyarthra vulgaris & $x$ & & \\
\hline Brachionus calyciflorus f. Amphicerus & $x$ & & & Scaridium sp. & $x$ & & $\mathrm{X}$ \\
\hline Brachionus dolabratus & $x$ & $\mathrm{X}$ & $\mathrm{X}$ & Synchaeta sp. & & $\mathrm{X}$ & $x$ \\
\hline Brachionus falcatus & & $x$ & $x$ & Testudinela ohlei & $X$ & $x$ & $\mathrm{X}$ \\
\hline Brachionus mirus & & $x$ & $x$ & Testudinella ah/strom & $x$ & & $\mathrm{x}$ \\
\hline Brachionus mirus angustus & $\mathrm{X}$ & & & Testudinella emarginula & $\mathrm{X}$ & & \\
\hline Brachionus mirus laticaudatus & $\mathrm{x}$ & & & Testudinella mucronata & $x$ & & $\mathrm{X}$ \\
\hline Brachionus mirus mirus & $\mathrm{X}$ & & & Testudinella ohlei & $x$ & & \\
\hline Brachionus quadridentatus quadridentatus & & & $\mathrm{X}$ & Testudinella patina & $x$ & $\mathrm{X}$ & $\mathrm{X}$ \\
\hline Brachionus tropica & & $\mathrm{X}$ & & Testudinella sp. & $\mathrm{X}$ & & $\mathrm{X}$ \\
\hline Brachionus zahniseri & $\mathrm{X}$ & $\mathrm{X}$ & $\mathrm{X}$ & Testudinella tridentata & $\mathrm{X}$ & & $\mathrm{X}$ \\
\hline Conochilus coenobasis & $x$ & & & Trichcocerca sp. & $x$ & $\mathrm{X}$ & $x$ \\
\hline Conochilus dossuarius & $x$ & & $\mathrm{X}$ & Trichocerca mus & & & $x$ \\
\hline Conochilus sp. & $x$ & $\mathrm{X}$ & $x$ & Trichotia tetractis & $\mathrm{X}$ & $x$ & $\mathrm{X}$ \\
\hline Dipleuchlanis propatula & $\mathrm{x}$ & & $x$ & Copepods & BF & DU & AF \\
\hline Fiinia saltator & & & $x$ & Argyrodiaptomus robertsonae & & & $x$ \\
\hline Filina limnetica & $\mathrm{X}$ & $\mathrm{x}$ & $x$ & Attheyella sp. & $x$ & & \\
\hline Filinia longiseta & $\mathrm{X}$ & $\mathrm{x}$ & & Calanoida & $x$ & $\mathrm{X}$ & $x$ \\
\hline Filinia opolienis & $x$ & $x$ & $\mathrm{X}$ & Cyclopoida & $x$ & $x$ & $x$ \\
\hline Filinia saltator & $x$ & $x$ & $x$ & Copepodito & $x$ & $x$ & $x$ \\
\hline Filinia sp. & $x$ & & & Copepodito ciclopoida & $x$ & $x$ & $x$ \\
\hline Filinia terminalis & $x$ & $\mathrm{X}$ & $x$ & Harpacticoida & $x$ & $x$ & $x$ \\
\hline Flosculariidae & $x$ & & & Mesocyclops meridianus & $x$ & & \\
\hline Hexarthra intermedia brasiliensis & $x$ & & & Mesocyclops sp. & $x$ & & $x$ \\
\hline Hexarthra sp. & $x$ & $x$ & $x$ & Metacyclops sp. & $x$ & $\mathrm{X}$ & $x$ \\
\hline
\end{tabular}


Table 1. Continued...

\begin{tabular}{|c|c|c|c|c|c|c|c|}
\hline Rotifers & BF & DU & AF & Rotifers (...continuing...) & BF & DU & AF \\
\hline Keratella amerciana & $\mathrm{X}$ & $\mathrm{X}$ & $\mathrm{X}$ & Microcyclops sp. & $\mathrm{X}$ & $\mathrm{X}$ & $\mathrm{X}$ \\
\hline Keratella cochlearis & $x$ & $\mathrm{x}$ & $\mathrm{x}$ & Nauplio & $x$ & $x$ & $x$ \\
\hline Keratella lenzi & $x$ & $\mathrm{X}$ & $\mathrm{X}$ & Nauplio Calanoida & $x$ & $\mathrm{x}$ & $x$ \\
\hline Keratella tropica & $x$ & $x$ & $\mathrm{X}$ & Nauplio Cyclopoida & $\mathrm{X}$ & $\mathrm{X}$ & $\mathrm{X}$ \\
\hline Keratellla lenzi & & & $\mathrm{X}$ & Notodiapotmus sp. & $\mathrm{X}$ & $x$ & $\mathrm{X}$ \\
\hline Lecane amazonica & $x$ & $X$ & $\mathrm{X}$ & Notodiaptomus henseni & $\mathrm{X}$ & & \\
\hline Lecane bulla & $x$ & $\mathrm{X}$ & $\mathrm{X}$ & Odontodiaptomus sp. & & $X$ & \\
\hline Lecane bulla bulla & $x$ & $x$ & $\mathrm{X}$ & Paracyclops sp. & $X$ & & \\
\hline Lecane closterocerca & & & $\mathrm{X}$ & Parastenocaris fontinalis & $\mathrm{X}$ & & \\
\hline Lecane cornuta & $X$ & & & Thermocyclops minutus & $\mathrm{X}$ & $X$ & $\mathrm{X}$ \\
\hline Lecane cornuta & & & $\mathrm{X}$ & Thermocyclops sp. & $\mathrm{X}$ & $x$ & $\mathrm{X}$ \\
\hline Lecane curvicornis & $X$ & & & Cladocerans & BF & DU & AF \\
\hline Lecane curvicornis curvicornis & $x$ & $X$ & $\mathrm{X}$ & Acroperus harpae & & & $\mathrm{X}$ \\
\hline Lecane curvicornis nitida & & & $\mathrm{X}$ & Acroperus sp. & & & $\mathrm{X}$ \\
\hline Lecane elsa & & & $\mathrm{X}$ & Alona guttata & $X$ & & \\
\hline Lecane haliclysta & $X$ & $X$ & & Alona sp. & $\mathrm{x}$ & & $X$ \\
\hline Lecane hamata & & & $\mathrm{X}$ & Alonella dadayi & $\mathrm{X}$ & $\mathrm{X}$ & $x$ \\
\hline Lecane leontina & $\mathrm{X}$ & & $\mathrm{x}$ & Alonella sp. & $x$ & & \\
\hline Lecane limnetica & & & $\mathrm{x}$ & Bosmina longirostris & $x$ & $X$ & $\mathrm{X}$ \\
\hline Lecane ludwigii & $X$ & & $x$ & Bosmina cf. Longirostris & $x$ & & \\
\hline Lecane ludwigii $f$. Ohiensis & & $X$ & & Bosmina sp. & $\mathrm{X}$ & & \\
\hline Lecane ludwigii ludwigii & & $x$ & & Bosminopsis deiterrsi & $\mathrm{X}$ & $X$ & $x$ \\
\hline Lecane luna & & & $X$ & Camptocercus sp. & & & $x$ \\
\hline Lecane lunaris & $x$ & $X$ & $\mathrm{X}$ & Ceriodaphania cornuta & $X$ & $X$ & $x$ \\
\hline Lecane lunaris crenata & & & $\mathrm{X}$ & Ceriodaphnia quadrangula & $\mathrm{X}$ & & \\
\hline Lecane monostyla & $X$ & $X$ & $\mathrm{x}$ & Ceriodaphnia richardi & $x$ & & \\
\hline Lecane pyriformis & & $x$ & & Ceriodaphnia sp. & $x$ & $X$ & $x$ \\
\hline Lecane quadridentata & $X$ & & $X$ & Chydorus eurynotus & & & $\mathrm{X}$ \\
\hline Lecane signifera & & $X$ & $\mathrm{X}$ & Chydorus parvireticulatus & $X$ & & \\
\hline Lecane sp. & $X$ & $x$ & & Chydorus sp. & $X$ & & $X$ \\
\hline Lecane stichaea & $x$ & & & Chydorus sphaericus & & & $X$ \\
\hline Lecane subtilis & $\mathrm{X}$ & $X$ & & Daphnia gessneri & $X$ & $X$ & $X$ \\
\hline Lecane thienemanne & $X$ & & $\mathrm{X}$ & Daphnia sp. & $X$ & $X$ & $\mathrm{X}$ \\
\hline Lecane ungulata & & $X$ & $\mathrm{X}$ & Diaphanosoma birgei & $\mathrm{X}$ & & \\
\hline Lepadella benjamini & $x$ & $x$ & $\mathrm{X}$ & Diaphanosoma brachyurum & $\mathrm{X}$ & & \\
\hline Lepadella ovalis & & $x$ & $\mathrm{x}$ & Diaphanosoma sp & $x$ & $X$ & $X$ \\
\hline Lepadella sp. & $X$ & & & Disparalona dadayi & $X$ & & $X$ \\
\hline Lophocharis sp. & & & $\mathrm{X}$ & Disparalona hamata & & & $\mathrm{X}$ \\
\hline Macrochaetus collinsi & & & $\mathrm{X}$ & Disparalona sp. 1 & $X$ & & \\
\hline Macrochaetus sericus & & & $\mathrm{X}$ & Ephemeroporus hybridus & $X$ & & $X$ \\
\hline Manfredium eudactylota euchla & $x$ & & & Graptoleberis testudinaria & & & $x$ \\
\hline Monommata sp. & $\mathrm{X}$ & & & Ilyocriptus spinifer & $\mathrm{X}$ & & $X$ \\
\hline Mytilina macrocera & $X$ & & $\mathrm{X}$ & Ilyocryptus sp. & & & $X$ \\
\hline Mytilina mucronata & & & $\mathrm{X}$ & Kurzia latissima & $X$ & & $x$ \\
\hline Mytilina sp. & $X$ & & & Leydigiopsis curvirostris & & & $\mathrm{X}$ \\
\hline Mytilina ventralis & $X$ & & & Leydigiopsis sp. & $X$ & $X$ & $\mathrm{X}$ \\
\hline Platias leloupi f. Latiscapularis & $\mathrm{X}$ & & & Macrothrix sp. & $X$ & & \\
\hline Plationus patulus macracanthus & $\mathrm{X}$ & $X$ & $X$ & Macrothrix triserialis & $X$ & & $\mathrm{X}$ \\
\hline Plationus patulus patulus & $x$ & $x$ & $\mathrm{X}$ & Moinodaphnia sp. & & $\mathrm{X}$ & \\
\hline Platyas quadricornis & $X$ & & & Notoalona sculpta & $X$ & & $x$ \\
\hline Platyias cf. Leloupi & $\mathrm{X}$ & $X$ & $\mathrm{X}$ & Pseudochydorus globosus & $X$ & & \\
\hline Platyias quadricornis & $X$ & $X$ & $\mathrm{X}$ & Scapholeberis sp. & $X$ & & \\
\hline Pleosoma lenticulare & & & $\mathrm{X}$ & Simocephalus sp. & & & $\mathrm{X}$ \\
\hline
\end{tabular}


There were compositional changes according to PERMANOVA considering nearly all datasets (except for Cladocerans using abundance data, Table 2). In agreement, there were typical species from $\mathrm{DU}$ and $\mathrm{AF}$ periods (but not $\mathrm{BF}$ ), as identified in IndVal analysis (Table 3). Compositional changes are also visible in PCoA diagrams (Figure 3). It is possible to observe a continuum of changes from period $\mathrm{BF}$ to $\mathrm{AF}$ (except for abundance of Cladocerans, as stated above). However, it is clear that for most comparisons, the period AF is the most different (Figure 3).

The spatial organization of Rotifers (measured as a matrix of dissimilarities among sampling units in each period) was similar in two comparisons for turnover (expect occurrence between $\mathrm{BF}$ and AF; and between DU and AF), but not for nestedness

Table 2. Results from Permutational Multivariate Analysis of Variance (F statistics and P value are shown) applied in zooplankton data (for each zooplankton group separately, see methods) considering both abundance and occurrence data.

\begin{tabular}{llll}
\hline $\begin{array}{l}\text { Zooplankton } \\
\text { group }\end{array}$ & $\begin{array}{c}\text { Data } \\
\text { resolution }\end{array}$ & F & P \\
\hline Rotifers & Abundance & 3.526 & 0.001 \\
Rotifers & Occurrence & 2.896 & 0.002 \\
Copepods & Abundance & 9.772 & 0.001 \\
Copepods & Occurrence & 7.057 & 0.001 \\
Cladocerans & Abundance & 1.627 & 0.101 \\
Cladocerans & Occurrence & 2.736 & 0.007 \\
\hline
\end{tabular}

(Table 4). For Copepods, spatial organization differed for almost all comparisons and data resolutions; only nestedness between DU and AF for occurrence data were correlated (Table 4). Finally, Cladocerans had a similar spatial organization in two comparisons for turnover (except for abundance turnover between $\mathrm{BF}$ and $\mathrm{AF}$ ), but not for nestedness (Table 4).

Total variation of community dissimilarity also differed between periods, but depending on the data resolution and facet of beta diversity (turnover or nestedness, see Table 4). For all groups, compositional variation in nestedness increased during the formation of the reservoir lake, and decreased after the formation of the lake (both for abundance and occurrence, see Table 5 and also the size of multivariate dispersion in Figure 3). Compositional variation in turnover did not differ for any group and data resolution (Table 5).

\section{Discussion}

Our results show a clear and immediate effect of damming in zooplankton community composition. We expected that compositional changes would be less affected for micro-crustaceans. Even so, only for abundance of Cladocerans we could not reject the hypothesis that species composition was not changed. Particularly after the formation of the lake, the species composition was the most different; indicating that species composition immediately

Table 3. Typical taxon or taxa stage (when not possible to identify) for each period identified as significantly different from a null expectation in Indicator Value (IndVal) analysis. The Indicator value is shown for the significant species in each period: during (DU) and after (AF) the reservoir lake formation. There was no typical species identified before reservoir formation.

\begin{tabular}{clccc}
\hline \multicolumn{1}{c}{ Taxon or stage } & Period & IndVal & $\boldsymbol{P}$ \\
\hline Rotifers & Trichocerca sp. & DU & 0.763 & 0.047 \\
& Keratella coclearis & DU & 0.580 & 0.005 \\
Brachionus falcatus & DU & 0.574 & 0.034 \\
Asplanchna brightwellii & AF & 0.900 & 0.001 \\
Keratella americana & AF & 0.688 & 0.002 \\
Lecane amazonica & AF & 0.665 & 0.002 \\
Synchaeta sp. & AF & 0.597 & 0.003 \\
Lecane leontina & AF & 0.586 & 0.009 \\
Testudinella mucronata & AF & 0.549 & 0.006 \\
& Lecane elsa & AF & 0.500 & 0.003 \\
Testudinella tridentata & AF & 0.496 & 0.008 \\
Filinia opoliensis & AF & 0.470 & 0.046 \\
Odontodiaptomus sp. & DU & 0.400 & 0.027 \\
Copepodito & DU & 0.378 & 0.039 \\
Cyclopoida & AF & 0.900 & 0.001 \\
Mesocyclops sp. & AF & 0.589 & 0.002 \\
Nauplio Cyclopoida & AF & 0.500 & 0.008 \\
Nauplio Calanoida & AF & 0.400 & 0.018 \\
\hline
\end{tabular}



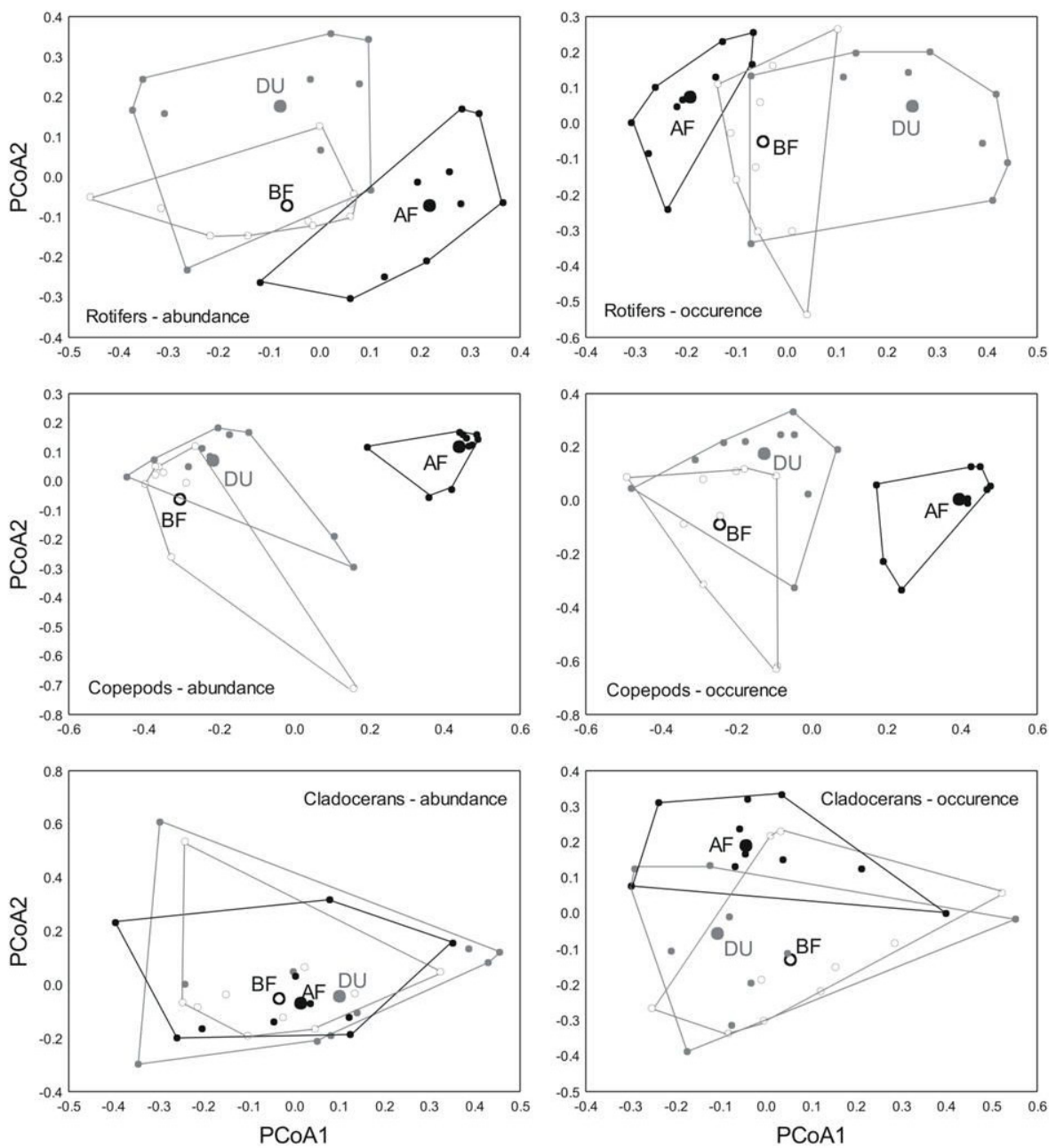

Figure 3. Two first axes of a Principal Coordinate Analysis (PCoA1 and PCoA2) showing the local communities (small balls) and the centroid (large balls) of each period of the dam construction (see methods) for abundance and occurrence of Rotifers, Copepods and Cladocerans. Before dam construction: BF, open light-black balls and light-black lines; During dam construction: DU, grey balls grey lines; After dam construction: AF, filled dark-black balls, dark-black lines.

Table 4. Mantel tests ( $\mathrm{r}$ and $\mathrm{P}$ ) correlating the spatial organization of communities between periods. Spatial organization was measured as a dissimilarity matrix between sampling units, which was estimated using turnover and nestedness components of beta diversity, both for abundance and for occurrence data, following Baselga (2010).

\begin{tabular}{cccccc}
\hline & Data Resolution & $\begin{array}{c}\text { Facet of beta } \\
\text { diversity }\end{array}$ & Comparison & Mantel's r & P \\
\hline Rotifers & Abundance & Turnover & BF-DU & $\mathbf{0 . 5 8 8}$ & $\mathbf{0 . 0 0 1}$ \\
& Abundance & Turnover & BF-AF & 0.406 & 0.036 \\
Abundance & Turnover & DU-AF & $\mathbf{0 . 6 2 6}$ & $\mathbf{0 . 0 0 1}$ \\
Abundance & Nestedness & BF-DU & -0.172 & 0.888 \\
Abundance & Nestedness & BF-AF & -0.353 & 0.994 \\
Abundance & Nestedness & DU-AF & -0.058 & 0.636 \\
Occurrence & Turnover & BF-DU & 0.388 & 0.039 \\
Occurrence & Turnover & BF-AF & 0.286 & 0.077 \\
Occurrence & Turnover & DU-AF & 0.369 & 0.077 \\
Occurrence & Nestedness & BF-DU & -0.239 & 0.940 \\
Occurrence & Nestedness & BF-AF & -0.345 & 0.999 \\
Occurrence & Nestedness & DU-AF & -0.017 & 0.551 \\
\hline
\end{tabular}

Comparisons were always made between pair of periods: $\mathrm{BF}=$ before; $\mathrm{DU}=$ during; and $\mathrm{AF}=$ after formation of the reservoir lake (see methods). Given that two comparisons were always done with a same dataset, we considered significant only when P values were lower than $2.5 \%$ (see methods). Significant values are highlighted in bold. 
Table 4. Continued...

\begin{tabular}{cccccc}
\hline & Data Resolution & $\begin{array}{c}\text { Facet of beta } \\
\text { diversity }\end{array}$ & Comparison & Mantel's r & P \\
\hline Copepods & Abundance & Turnover & BF-DU & -0.139 & 0.696 \\
& Abundance & Turnover & BF-AF & 0.139 & 0.184 \\
& Abundance & Turnover & DU-AF & -0.160 & 0.743 \\
& Abundance & Nestedness & BF-DU & -0.068 & 0.612 \\
& Abundance & Nestedness & BF-AF & 0.025 & 0.442 \\
& Abundance & Nestedness & DU-AF & 0.217 & 0.140 \\
& Occurrence & Turnover & BF-DU & 0.013 & 0.447 \\
& Occurrence & Turnover & BF-AF & 0.062 & 0.352 \\
& Occurrence & Turnover & DU-AF & -0.190 & 0.772 \\
Occurrence & Nestedness & BF-DU & 0.066 & 0.322 \\
Occurrence & Nestedness & BF-AF & 0.243 & 0.053 \\
Occurrence & Nestedness & DU-AF & $\mathbf{0 . 4 7 6}$ & $\mathbf{0 . 0 1 6}$ \\
Abundance & Turnover & BF-DU & 0.610 & 0.049 \\
Abundance & Turnover & BF-AF & 0.412 & 0.124 \\
& Abundance & Turnover & DU-AF & $\mathbf{0 . 5 8 1}$ & $\mathbf{0 . 0 0 7}$ \\
Abundance & Nestedness & BF-DU & 0.016 & 0.467 \\
Abundance & Nestedness & BF-AF & -0.199 & 0.818 \\
Abundance & Nestedness & DU-AF & 0.072 & 0.337 \\
Occurrence & Turnover & BF-DU & 0.421 & 0.045 \\
Occurrence & Turnover & BF-AF & $\mathbf{0 . 7 0 2}$ & $\mathbf{0 . 0 0 2}$ \\
Occurrence & Turnover & DU-AF & $\mathbf{0 . 3 7 0}$ & $\mathbf{0 . 0 1 8}$ \\
Occurrence & Nestedness & BF-DU & -0.063 & 0.588 \\
Occurrence & Nestedness & BF-AF & 0.119 & 0.246 \\
Occurrence & Nestedness & DU-AF & -0.021 & 0.517 \\
\hline
\end{tabular}

Comparisons were always made between pair of periods: $\mathrm{BF}$ = before; $\mathrm{DU}=$ during; and $\mathrm{AF}$ = after formation of the reservoir lake (see methods). Given that two comparisons were always done with a same dataset, we considered significant only when P values were lower than $2.5 \%$ (see methods). Significant values are highlighted in bold.

Table 5. Variation in compositional dissimilarity (calculated following betadisper approach, see Anderson et al., 2006) for turnover and nestedness (following Baselga, 2010) using both abundance and occurrence data for each zooplankton group estimated before (BF), during (DU) and after (AF) the formation of the reservoir (see methods). The F statistics and P value for the permutation test is also shown. Significant values are highlighted in bold.

\begin{tabular}{cccccc}
\hline & Data Resolution & Period & $\begin{array}{c}\text { Facet of beta } \\
\text { diversity }\end{array}$ & $\begin{array}{c}\text { Variation in } \\
\text { compositional } \\
\text { dissimilarity }\end{array}$ & $\begin{array}{c}\text { Permutation } \\
\text { test }\end{array}$ \\
\hline Rotifers & Abundance & BF & Turnover & 0.402 & $\mathrm{~F}=0.904$ \\
& Abundance & DU & Turnover & 0.332 & $\mathrm{P}=0.425$ \\
& Abundance & $\mathrm{AF}$ & Turnover & 0.349 & \\
& Abundance & $\mathrm{BF}$ & Nestedness & 0.034 & $\mathrm{~F}=\mathbf{5 . 0 5 7}$ \\
& Abundance & $\mathrm{DU}$ & Nestedness & 0.064 & $\mathrm{P}=\mathbf{0 . 0 1 4}$ \\
& Abundance & $\mathrm{AF}$ & Nestedness & 0.030 & \\
& Occurrence & $\mathrm{BF}$ & Turnover & 0.393 & $\mathrm{~F}=3.147$ \\
& Occurrence & $\mathrm{DU}$ & Turnover & 0.243 & $\mathrm{P}=0.057$ \\
Occurrence & $\mathrm{AF}$ & Turnover & 0.345 & \\
& Occurrence & $\mathrm{BF}$ & Nestedness & 0.110 & $\mathrm{~F}=\mathbf{1 3 . 6 4 3}$ \\
& Occurrence & $\mathrm{DU}$ & Nestedness & 0.246 & $\mathrm{P}=\mathbf{0 . 0 0 2}$ \\
& Occurrence & $\mathrm{AF}$ & Nestedness & 0.097 & \\
& Abundance & $\mathrm{BF}$ & Turnover & 0.390 & $\mathrm{~F}=0.028$ \\
& Abundance & $\mathrm{DU}$ & Turnover & 0.386 & $\mathrm{P}=0.968$ \\
& Abundance & $\mathrm{AF}$ & Turnover & 0.409 & \\
& Abundance & $\mathrm{BF}$ & Nestedness & 0.022 & $\mathrm{~F}=\mathbf{7 . 5 9 3}$ \\
& Abundance & $\mathrm{DU}$ & Nestedness & 0.070 & $\mathrm{P}=\mathbf{0 . 0 0 1}$ \\
& Abundance & $\mathrm{AF}$ & Nestedness & 0.016 & \\
& Occurrence & $\mathrm{BF}$ & Turnover & 0.390 & $\mathrm{~F}=1.301$ \\
& Occurrence & $\mathrm{DU}$ & Turnover & 0.386 & $\mathrm{P}=0.291$ \\
& Occurrence & $\mathrm{AF}$ & Turnover & 0.409 & \\
& Occurrence & $\mathrm{BF}$ & Nestedness & 0.022 & $\mathrm{~F}=\mathbf{3 . 1 1 9}$ \\
Occurrence & $\mathrm{DU}$ & Nestedness & 0.070 & $\mathrm{P}=\mathbf{0 . 0 2 9}$ \\
Occurrence & $\mathrm{AF}$ & Nestedness & 0.016 & \\
\hline
\end{tabular}


Table 5. Continued...

\begin{tabular}{|c|c|c|c|c|c|}
\hline & Data Resolution & Period & $\begin{array}{l}\text { Facet of beta } \\
\text { diversity }\end{array}$ & $\begin{array}{c}\text { Variation in } \\
\text { compositional } \\
\text { dissimilarity }\end{array}$ & $\begin{array}{c}\text { Permutation } \\
\text { test }\end{array}$ \\
\hline \multirow[t]{12}{*}{ Cladocerans } & Abundance & $\mathrm{BF}$ & Turnover & 0.377 & \multirow{3}{*}{$\begin{array}{l}F=0.149 \\
P=0.887\end{array}$} \\
\hline & Abundance & DU & Turnover & 0.326 & \\
\hline & Abundance & $\mathrm{AF}$ & Turnover & 0.359 & \\
\hline & Abundance & $\mathrm{BF}$ & Nestedness & 0.023 & \multirow{3}{*}{$\begin{array}{l}F=4.108 \\
P=0.024\end{array}$} \\
\hline & Abundance & DU & Nestedness & 0.055 & \\
\hline & Abundance & AF & Nestedness & 0.033 & \\
\hline & Occurrence & $\mathrm{BF}$ & Turnover & 0.377 & \multirow{3}{*}{$\begin{array}{l}F=1.729 \\
P=0.193\end{array}$} \\
\hline & Occurrence & DU & Turnover & 0.326 & \\
\hline & Occurrence & $\mathrm{AF}$ & Turnover & 0.359 & \\
\hline & Occurrence & $\mathrm{BF}$ & Nestedness & 0.023 & \multirow{3}{*}{$\begin{array}{l}F=6.017 \\
P=0.006\end{array}$} \\
\hline & Occurrence & DU & Nestedness & 0.055 & \\
\hline & Occurrence & $A F$ & Nestedness & 0.033 & \\
\hline
\end{tabular}

changes with landscape changes. More than showing the well-described pattern of compositional changes due to reservoir damming (e.g. Simóes et al., 2015) we also indicate that changes are directional, promoting certain species during and after the formation of the lake. Just after the formation of the lake, water is usually turbid and nutrient-rich due to the decomposition of remnant vegetation, promoting cyanobacteria (Kennedy \& Thornton, 2001). Among the typical zooplankton species found in this period are Asplanchna brightwellii and a Cyclopoid species. Also, our results are in line with previous studies indicating that species from Lecanidae, Brachionidae and Trichocercidae are typical after floods, where habitat connection is higher and community similarity is greater (Bonecker et al., 2013).

Our study goes further in describing the impact of dam construction by showing that effects in composition occurs for both abundance and occurrence data. Even so, we do highlight that such analyses should be done with both data resolutions, given that impact was identified for Cladocerans only using occurrence data. Probably, the fact that most abundance species occurred in all periods, compositional changes using abundance data is more difficult to identify. We thus refute the suggestion that a significant correlation between abundance and occurrence data could simplify monitoring (e.g. Ribas \& Padial, 2015; Souza et al., 2019). At least for impact assessment, we highlight that analyzing different data resolutions should be preferred.

We also suggest that impact of dams also disrupt spatial community organization, which suggest that species filtering mechanisms (Leibold et al., 2004) are also altered by reservoir damming.
Indeed, responses of zooplankton to environmental gradients have been altered by reservoir damming (Portinho et al., 2016). Again, we reinforce that a complete investigation should be done, given that such patter was described for most but not all comparisons (see Table 3). The fact that Rotifers are more affected by both compositional changes as well as for changes in spatial organization of metacommunity was expected, as this group is more susceptible to environmental alterations and is more sessile (Soares et al., 2015). However, our results also suggest that Cladocerans can also have a disruption of its spatial organization, but the fact that effect was detected using occurrence may suggest that the disruption occurred mainly in less abundance taxa, which could be more susceptible to environmental alterations promoted by reservoir damming. Relatedly, Missias et al. (2017) also suggest that Rotifers, Copepods and Cladocerans do respond differently to spatial ecological gradients in reservoirs; which reinforce that all groups should be evaluated separately in studies evaluating anthropogenic impacts in aquatic ecosystems.

Contrarily to what we expected, reservoirs did not necessarily decreased compositional variation over space; which would indicate a biotic homogenization phenomenon (Olden \& Rooney, 2006); already evidenced in other reservoirs (Daga et al., 2015). Maybe the homogenization phenomenon could still occur, given that such phenomenon does need a long time-spam to be identified (see Olden et al., 2018). However, by scrutinizing data and evaluating turnover and nestedness separately, our results do show an interesting pattern: an increase in nestedness during the formation of the lake. We did not find any report on this in literature. Instead, Lopes et al. (2017) 
suggested that temporal variation of zooplankton beta diversity components are difficult to predict and do not have a monotonic pattern of increase and decrease in a tropical reservoir. However, such study took place only after the formation of the reservoir. The increase in nestedness during the formation of the lake do indicate that is this period, the metacommunity experience a relatively more impacted gradient. Indeed, increasing nestedness is associated to an ecological gradient of impact (e.g. Declerck et al., 2007). In our study, the increase in nestedness occurred for all biological groups and data resolutions. Even so, we do recognize that impacts were not overwhelming compared to others that may combine high turnover with increasing nestedness.

In this study, we did show the trajectory of zooplankton community following to the formation of a reservoir lake. We highlight that impacts are detectable using different facets of metacommunity beta-diversity, including compositional changes, spatial organization changes and compositional variation changes (see Anderson et al., 2011 for a description of different ways to evaluate beta-diversity). We reinforce the urge in literature for a complete assessment of biodiversity (Socolar et al., 2016) and also for digging deeper in evaluating phenomena such as Biotic Homogenization as a consequence of anthropogenic impacts (Olden et al., 2018). We also suggest that data resolution and differential responses of biological groups are features that should be considered in zooplankton assessments (Missias et al., 2017). Our study is unique by following the trajectory of the formation of a reservoir lake in a still under-study aquatic biological group. We showed that zooplankton cope with landscape changes caused by reservoir lake formation, which may also reflect in ecosystem functioning features such as productivity, matter and nutrient cycling (Simóes et al., 2015). It is well-known that environmental alterations caused by changes associated to reservoirs extend to biota (Agostinho et al., 2008). Here, we scrutinized the complexity of effects in zooplankton, considering data resolution, biological groups and the facet of biodiversity evaluated. Given the relatively short time-spam (i.e. three years) studied; we do suggest that monitoring in this reservoir continues to evaluate the long-term effects that river-damming cause to zooplankton community. For instance, the compositional changes observed here can be only transitory, or other changes can be only identified after a long time-spam. It is also worth-mentioning that is not prudent to infer causality based on observational studies. An approach to do so is the so called 'counterfactual thinking' (Ribas et al., 2019). Therefore, we also highlight the need to monitor and preserve pristine riverine ecosystems to meet conservation of biodiversity and also to a better inference of causality in anthropogenic impacts (e.g. Ribas et al., 2019). We believe that monitoring such as the realized in this case is a model to be followed in other dam constructions, if simultaneously accompanied by monitoring in similar areas without impacts.

\section{Acknowledgements}

We are grateful to Dr. Victor Saito for insightful comments in a previous version of this manuscript. We acknowledge COPEL for making data available for scientific studies and Lactec institute for providing all logistic support for data sampling and identification, and for a scholarship granted to J. S. A.A.P. also acknowledges $\mathrm{CNPq}$ for continuous financial supports (current projects process numbers: 4022828/2016-0 and 301867/2018-6).

\section{References}

AGOSTINHO, A.A., PELICICE, F.M. and GOMES, L.C. Dams and the fish fauna of the Neotropical region: impacts and management related to diversity and fisheries. Brazilian Journal of Biology = Revista Brasileira de Biologia, 2008, 68(4, Suppl.), 1119-1132. http://dx.doi.org/10.1590/S151969842008000500019. PMid:19197482.

ANDERSON, M.J. A new method for non-parametric multivariate analysis of variance. Austral Ecology, 2001, 26(1), 32-46.

ANDERSON, M.J., CRIST, T.O., CHASE, J.M., VELLEND, M., INOUYE, B.D., FREESTONE, A.L., SANDERS, N.J., CORNELL, H.V., COMITA, L.S., DAVIES, K.F., HARRISON, S.P., KRAFT, N.J., STEGEN, J.C. and SWENSON, N.G. Navigating the multiple meanings of $\beta$ diversity: a roadmap for the practicing ecologist. Ecology Letters, 2011, 14(1), 19-28. http://dx.doi.org/10.1111/ j.1461-0248.2010.01552.x. PMid:21070562.

ANDERSON, M.J., ELLINGSEN, K.E. and MCARDLE, B.H. Multivariate dispersion as a measure of beta diversity. Ecology Letters, 2006, 9(6), 683-693. http://dx.doi.org/10.1111/j.14610248.2006.00926.x. PMid:16706913.

AZEVEDO-SANTOS, V.M., FEARNSIDE, P.M., OLIVEIRA, C.S., PADIAL, A.A., PELICICE, F.M., LIMA JUNIOR, D.P., SIMBERLOFF, D., LOVEJOY, T.E., MAGALHÁES, A.L.B., ORSI, M.L., AGOSTINHO, A.A., ESTEVES, F.A., POMPEU, P.S., LAURANCE, W.F., PETRERE 
JUNIOR, M., MORMUL, R.P. and VITULE, J.R.S. Removing the abyss between conservation science and policy decision in Brazil. Biodiversity and Conservation, 2017, 26(7), 1745-1752. http://dx.doi. org/10.1007/s10531-017-1316-x.

BASELGA, A. Partitioning the turnover and nestedness components of beta diversity. Global Ecology and Biogeography, 2010, 19(1), 134-143. http://dx.doi. org/10.1111/j.1466-8238.2009.00490.x.

BASELGA, A., ORME, D., VILLEGER, S., BORTOLI, J. and LEPRIEUR, F. betapart: partitioning beta diversity into turnover and nestedness components: $R$ package version 1.5.1. Vienna: R Foundation for Statistical Computing, 2018.

BAXTER, R.M. Environmental effects of reservoirs. In: D. GUNNISON, ed. Microbial processes in reservoirs. Dordrecht: Springer, 1985, pp. 1-26. Developments in Hydrobiology, vol. 27. http:// dx.doi.org/10.1007/978-94-009-5514-1_1.

BONECKER, C.C., SIMÓES, N.R., MINTE-VERA, C.V., LANSAC-TÔHA, F.A., VELHO, L.F.M. and AGOSTINHO, A.A. Temporal changes in zooplankton species diversity in response to environmental changes in an alluvial valley. Limnologica, 2013, 43(2), 114-121. http://dx.doi. org/10.1016/j.limno.2012.07.007.

BOZELLI, R.L., THOMAZ, S.M., PADIAL, A.A., LOPES, P.M. and BINI, L.M. Floods decrease zooplankton beta diversity and environmental heterogeneity in an Amazonian floodplain system. Hydrobiologia, 2015, 753(1), 233-241. http://dx.doi. org/10.1007/s10750-015-2209-1.

COMPANHIA PARANAENSE DE ENERGIA COPEL. Hydroelectric Power Plant de Colíder [online]. Curitiba: COPEL, 2018 [viewed 5 Sept. 2018]. Available from: http://www.copel.com/ uhecolider/

COUTO, T.B.A. and OLDEN, J.D. Global proliferation of small hydropower plants - science and policy. Frontiers in Ecology and the Environment, 2018, 16(2), 91-100. http://dx.doi.org/10.1002/fee.1746.

DAGA, V.S., SKÓRA, F., PADIAL, A.A., ABILHOA, V., GUBIANI, E.A. and VITULE, J.R.S. Homogenization dynamics of the fish assemblages in Neotropical reservoirs: Comparing the roles of introduced species and their vectors. Hydrobiologia, 2015, 746(1), 327-347. http://dx.doi.org/10.1007/ s10750-014-2032-0.

DECLERCK, S., VANDERSTUKKEN, M., PALS, A., MUYLAERT, K. and MEESTER, L.D. Plankton biodiversity along a gradient of productivity and its mediation by macrophytes. Ecology, 2007, 88(9), 2199-2210. http://dx.doi.org/10.1890/07-0048.1. PMid:17918398.

DUFRENE, M. and LEGENDRE, P. Species assemblages and indicator species: the need for a flexible asymmetrical approach. Ecological Monographs, 1997, 67(3), 345-366. http://dx.doi.org/10.2307/2963459.

ELMOOR-LOUREIRO, L.M.A. Manual de identificação de Cladóceros límnicos do Brasil. Brasília: Universidade Católica de Brasília, 1997.

GAZULHA, V. Zooplâncton límnico: manual ilustrado. 1. ed. Rio de Janeiro: Technical Books, 2012.

GOWER, J.C. Some distance properties of latent root and vector methods used in multivariate analysis. Biometrika, 1966, 53(3-4), 325-328. http://dx.doi. org/10.1093/biomet/53.3-4.325.

JOKO, C. Y. Taxonomia de Rotiferos monogonontas da planície de inundação do alto rio Paraná (MS/PR) [Tese de Doutorado em Ecologia de Ambientes Aquáticos Continentais]. Maringá: Departamento de Biologia, Universidade Estadual de Maringá, 2011.

KENNEDY, R.H. and THORNTON, K.W. Water quality indicators for reservoirs: a conceptual framework. Lake and Reservoir Management, 2001, 17(3), 188-196. http://dx.doi. org/10.1080/07438140109354130.

LEGENDRE, P. and GALLAGHER, E.D. Ecologically meaningful transformations for ordination of species data. Oecologia, 2001, 129(2), 271-280. http://dx.doi. org/10.1007/s004420100716. PMid:28547606.

LEIBOLD, M.A., HOLYOAK, M., MOUQUET, N., AMARASEKARE, P., CHASE, J.M., HOOPES, M.F., HOLT, R.D., SHURIN, J.B., LAW, R., TILMAN, D., LOREAU, M. and GONZALEZ, A. The metacommunity concept: a framework for multi-scale community ecology. Ecology Letters, 2004, 7(7), 601-613. http://dx.doi.org/10.1111/j.14610248.2004.00608.x.

LOPES, V.G., CASTELO BRANCO, C.W., KOZLOWSKY-SUZUKI, B., SOUSA-FILHO, I.F., SOUZA, L.C. and BINI, L.M. Predicting temporal variation in zooplankton beta diversity is challenging. PLoS One, 2017, 12(11), e0187499. http://dx.doi.org/10.1371/journal.pone.0187499. PMid:29095892.

MANTEL, N. The detection of disease clustering and a generalized regression approach. Cancer Research, 1967, 27(2), 209-220. PMid:6018555.

MISSIAS, A.C.A., GOMES, L.F., PEREIRA, H.R., SILVA, L.C.F., ANGELINI, R. and VIEIRA, L.C.G. Is it possible to simplify environmental monitoring? Approaches with zooplankton in a hydroelectric reservoir. Acta Limnologica Brasiliensia, 2017, 29, e8. http://dx.doi.org/10.1590/s2179-975x6516.

NOLETO, E.V., BARBOSA, M.V.M. and PELICICE, F.M. Distribution of aquatic macrophytes along depth gradients in Lajeado Reservoir, Tocantins River, Brazil. Acta Limnologica Brasiliensia, 2019, 31, e6. http://dx.doi.org/10.1590/s2179-975x9317.

OKSANEN, J., BLANCHET, F. G., FRIENDLY M., KINDT R., LEGENDRE P., MCGLINN, D., 
MINCHIN, P. R., O'HARA, R. B., SIMPSON, G. L., SOLYMOS, P., STEVENS, M. H. H., SZOECS, E., and WAGNER, H. R CRAN: package vegan: community ecology package. Vienna: R Foundation for Statistical Computing, 2017.

OLDEN, J.D. and ROONEY, T.P. On defining and quantifying biotic homogenization. Global Ecology and Biogeography, 2006, 15(2), 113-120. http:// dx.doi.org/10.1111/j.1466-822X.2006.00214.x.

OLDEN, J.D., COMTE, L. and GIAM, X. The Homogocene: a research prospectus for the study of biotic homogenisation. NeoBiota, 2018, 37, 23-36. http://dx.doi.org/10.3897/neobiota.37.22552.

PELICICE, F.M., POMPEU, P.S. and AGOSTINHO, A.A. Large reservoirs as ecological barriers to downstream movements of Neotropical migratory fish. Fish and Fisheries, 2015, 16(4), 697-715. http:// dx.doi.org/10.1111/faf.12089.

PORTINHO, J.L., PERBICHE-NEVES, G. and NOGUEIRA, M.G. Zooplankton community and tributary effects in free-flowing section downstream a large tropical reservoir. International Review of Hydrobiology, 2016, 101(1-2), 48-56. http://dx.doi. org/10.1002/iroh.201501798.

R CORETEAM. (2017). R: a language and environment for statistical computing [online]. Vienna: R Foundation for Statistical Computing. [viewed 5 Sept. 2018]. Available from: https://www.R-project.org/

RIBAS, L.G.S. and PADIAL, A.A. The use of coarser data is an effective strategy for biological assessments. Hydrobiologia, 2015, 747(1), 83-95. http://dx.doi. org/10.1007/s10750-014-2128-6.

RIBAS, L.G.S., PADIAL, A.A. and BINI, L.M. Avançando em avaliaçóes de impacto na limnologia aplicada. Acta Limnologica Brasiliensia, 2019, 31, e101. http://dx.doi.org/10.1590/s2179-975x2119.

ROBERTS, D. W. R CRAN: package labdsv: ordination and multivariate analysis for ecology. Vienna: $\mathrm{R}$ Foundation for Statistical Computing, 2016.

RUTTNER-KOLISKO, A. Plankton rotifers: biology and taxonomy. Stuttgart: Schweizerbart, 1974.

SHIEL, R.J. A guide to identification of rotifers, cladocerans and copepods from Australian inland waters. Bruce, Australia: Cooperative Research Centre for Freshwater Ecology, 1995. Identification Guide, vol. 3.

SILVA, E.N.S., ROBERTSON, B.A., REID, J.L.W. and HARDY, E.R. Atlas of planktonic Copepods, Calanoida and cyclopoida (Crustacea) from the Brazilian Amazon. I. Curuá-Dam Dam, Para. Revista Brasileira de Zoologia, 1989, 6(4), 725-758. http:// dx.doi.org/10.1590/S0101-81751989000400019.

SILVA, J.C., ROSA, R.R., GALDIOLI, E.M., SOARES, C.M., DOMINGUES, W.M., VERÍSSIMO, S. and BIALETZKI, A. Importance of dam-free stretches for fish reproduction: the last remnant in the Upper
Paraná River. Acta Limnologica Brasiliensia, 2017, 29(0), e106. http://dx.doi.org/10.1590/s2179$975 \times 10216$.

SIMÓES, N.R., NUNES, A.H., DIAS, J.D., LANSACTÔHA, F.A., VELHO, L.F.M. and BONECKER, C.C. Impact of reservoirs on zooplankton diversity and implications for the conservation of natural aquatic environments. Hydrobiologia, 2015, 758(1), 3-17. http://dx.doi.org/10.1007/s10750-015$2260-y$.

SOARES, C.E.A., VELHO, L.F.M., LANSAC-TÔHA, F.A., BONECKER, C.C., LANDEIRO, V.L. and BINI, L.M. The likely effects of river impoundment on beta-diversity of a floodplain zooplankton metacommunity. Natureza \& Conservação, 2015, 13(1), 74-79. http://dx.doi.org/10.1016/j. ncon.2015.04.002.

SOCOLAR, J.B., GILROY, J.J., KUNIN, W.E. and EDWARDS, D.P. How should beta-diversity inform biodiversity conservation? Trends in Ecology \& Evolution, 2016, 31(1), 67-80. http://dx.doi. org/10.1016/j.tree.2015.11.005. PMid:26701706.

SOUZA, C.A., MACHADO, K.B., NABOUT, J.C., MUNIZ, D.H.F., OLIVEIRA-FILHO, E.C., KRAUS, C.N., RIBEIRO, R.J.C. and VIEIRA, L.C.G. Monitoring simplification in plankton communities using different ecological approaches. Acta Limnologica Brasiliensia, 2019, 31, e20. http:// dx.doi.org/10.1590/s2179-975x3617.

VIEIRA, M.C., ROITMAN, I., BARBOSA, H.O., VELHO, L.F.M. and VIEIRA, L.C.G. Spatial synchrony of zooplankton during the impoundment of amazonic reservoir. Ecological Indicators, 2019, 98, 649-656. http://dx.doi.org/10.1016/j. ecolind.2018.11.040.

VINCENTIN, A.M., RODRIGUES, E.H.C., MOSCHINI-CARLOS, V. and POMPÊ, M.L.M. Is it possible to evaluate the ecological status of a reservoir using the phytoplankton community? Acta Limnologica Brasiliensia, 2018, 30, e306.

VON SPERLING, E. Hydropower in Brazil: overview of positive and negative environmental aspects. Energy Procedia, 2012, 18, 110-118. http://dx.doi. org/10.1016/j.egypro.2012.05.023.

WINEMILLER, K.O., MCINTYRE, P.B., CASTELLO, L., FLUET-CHOUINARD, E., GIARRIZZO, T., NAM, S., BAIRD, I.G., DARWALL, W., LUJAN, N.K., HARRISON, I., STIASSNY, M.L.J., SILVANO, R.A.M., FITZGERALD, D.B., PELICICE, F.M., AGOSTINHO, A.A., GOMES, L.C., ALBERT, J.S., BARAN, E., PETRERE JUNIOR, M., ZARFL, C., MULLIGAN, M., SULLIVAN, J.P., ARANTES, C.C., SOUSA, L.M., KONING, A.A., HOEINGHAUS, D.J., SABAJ, M., LUNDBERG, J.G., ARMBRUSTER, J., THIEME, M.L., PETRY, P., ZUANON, J., VILARA, G.T., SNOEKS, J., OU, C., RAINBOTH, W., 
PAVANELLI, C.S., AKAMA, A., SOESBERGEN, A. and SAENZ, L. Balancing hydropower and biodiversity in the Amazon, Congo, and Mekong. Science, 2016, 351(6269), 128-129. http://dx.doi. org/10.1126/science.aac7082. PMid:26744397.

WITTY, L.M. Practical guide to Identifying freshwater crustacean zooplankton. 2nd ed. Sudbury: Cooperative Freshwater Ecology Unit Department of Biology, 2004.

WOSIACK, A. C., ARRUDA, N. M. B., PIRES, G. R. M., SIECIECHOWICZ, M. S. F., TREMARIN, P. I., ANDRADE, P. D. B., and LARCHER, L. Diagnóstico das condiçôes limnológicas e da qualidade da água superficial na região do empreendimento UHE Colider: relatório 6 - Ciclo 2016-2017. Curitiba: COPEL, 2018.

ZHAO, K., SONG, K., PAN, Y., WANG, L., DA, L. and WANG, Q. Metacommunity structure of zooplankton in river networks: roles of environmental and spatial factors. Ecological Indicators, 2017, 73, 96-104. http://dx.doi.org/10.1016/j.ecolind.2016.07.026.

Received: 24 October 2019 Accepted: 14 April 2020

Associate Editor: Victor Satoru Saito. 


\title{
ERRATUM: Zooplankton trajectory before, during and after a hydropower dam construction
}

\author{
ERRATUM: Trajetória do zooplâncton antes, durante e depois da construção de uma \\ barragem de hidroelétrica
}

\section{Jaqueline Schmidt ${ }^{1}$ (D), Patrícia Dammski Borges de Andrade ${ }^{2}$ (D) and}

$$
\text { André Andrian Padial }{ }^{1 *} \text { (D) }
$$

\footnotetext{
${ }^{1}$ Laboratório de Análise e Síntese em Biodiversidade, Departamento de Botânica, Setor de Ciências Biológicas, Universidade Federal do Paraná - UFPR, Av. Cel Francisco Heráclito dos Santos, s/n, Jardim das Américas, CEP 81531-990, Curitiba, PR, Brasil

${ }^{2}$ LACTEC Instituto de Tecnologia para o Desenvolvimento, Rodovia BR-116, Km 98, 8813,

Prédio CEHPAR, Jardim das Américas, CEP 81531-980, Curitiba, PR, Brasil

*e-mail: aapadial@gmail.com
}

In the article "Zooplankton trajectory before, during and after a hydropower dam construction", DOI: https://doi.org/10.1590/S2179-975X9519, published in Acta Limnologica Brasiliensia, 2020, vol. 32, e18:

Authors are really sorry for some spelling mistakes in species names in Tables 1 and 3. See below the correct tables. Also, authors used capital letters to refer for biological groups along the text, such as 'Rotifers', 'Cladocerans' and 'Copepods'. Authors should use lowercase letters in these cases and keep capital letters only for the formal names of taxonomic categories.

Were it reads:

Table 1. Full list of zooplankton taxa (separated by Rotifers, Copepods and Cladocerans) sampled before (BF), during (DU) and after (AF) the formation of the reservoir lake from the hydropower dam UHE Colíder.

\begin{tabular}{|c|c|c|c|c|c|c|c|}
\hline Rotifers & BF & DU & AF & Rotifers (...continuing...) & $\mathrm{BF}$ & DU & AF \\
\hline Ascomorpha sp. & $\mathrm{x}$ & $\mathrm{X}$ & $\mathrm{x}$ & Pleosoma sp. & $\mathrm{X}$ & & \\
\hline Asplanchna brightwellii & & & $\mathrm{x}$ & Ploesoma truncatum & & & $\mathrm{x}$ \\
\hline Asplanchna sieboldii & $\mathrm{x}$ & & & Polyarthra dolichoptera & $\mathrm{x}$ & & \\
\hline Asplanchna sp. & $x$ & $\mathrm{x}$ & $\mathrm{x}$ & Polyarthra remata & $x$ & & \\
\hline Bdelloidea & $x$ & $x$ & $x$ & Polyarthra sp. & $x$ & $\mathrm{x}$ & $\mathrm{x}$ \\
\hline Beauchampiella sp. & $\mathrm{x}$ & & $\mathrm{x}$ & Polyarthra vulgaris & $\mathrm{x}$ & & \\
\hline Brachionus calyciflorus f. Amphicerus & $x$ & & & Scaridium sp. & $x$ & & $\mathrm{x}$ \\
\hline Brachionus dolabratus & $x$ & $\mathrm{x}$ & $\mathrm{x}$ & Synchaeta sp. & & $\mathrm{x}$ & $x$ \\
\hline Brachionus falcatus & & $\mathrm{x}$ & $\mathrm{x}$ & Testudinela ohlei & $\mathrm{x}$ & $\mathrm{x}$ & $\mathrm{x}$ \\
\hline Brachionus mirus & & $\mathrm{x}$ & $\mathrm{x}$ & Testudinella ahlstrom & $\mathrm{x}$ & & $\mathrm{x}$ \\
\hline Brachionus mirus angustus & $\mathrm{x}$ & & & Testudinella emarginula & $x$ & & \\
\hline Brachionus mirus laticaudatus & $x$ & & & Testudinella mucronata & $x$ & & $\mathrm{x}$ \\
\hline Brachionus mirus mirus & $x$ & & & Testudinella ohlei & $x$ & & \\
\hline $\begin{array}{l}\text { Brachionus quadridentatus } \\
\text { quadridentatus }\end{array}$ & & & $x$ & Testudinella patina & $x$ & $\mathrm{x}$ & $x$ \\
\hline Brachionus tropica & & $\mathrm{x}$ & & Testudinella sp. & $\mathrm{x}$ & & $\mathrm{x}$ \\
\hline
\end{tabular}


Table 1. Continued...

\begin{tabular}{|c|c|c|c|c|c|c|c|}
\hline Rotifers & BF & DU & AF & Rotifers (...continuing...) & BF & DU & AF \\
\hline Brachionus zahniseri & $\mathrm{X}$ & $X$ & $x$ & Testudinella tridentata & $X$ & & $\mathrm{X}$ \\
\hline Conochilus coenobasis & $x$ & & & Trichcocerca sp. & $x$ & $x$ & $\mathrm{X}$ \\
\hline Conochilus dossuarius & $x$ & & $x$ & Trichocerca mus & & & $\mathrm{X}$ \\
\hline Conochilus sp. & $x$ & $x$ & $x$ & Trichotia tetractis & $x$ & $x$ & $x$ \\
\hline Dipleuchlanis propatula & $x$ & & $x$ & Copepods & $\mathrm{BF}$ & DU & $\mathrm{AF}$ \\
\hline Fiinia saltator & & & $x$ & Argyrodiaptomus robertsonae & & & $x$ \\
\hline Filina limnetica & $X$ & $\mathrm{X}$ & $x$ & Attheyella sp. & $\mathrm{X}$ & & \\
\hline Filinia longiseta & $x$ & $x$ & & Calanoida & $x$ & $x$ & $X$ \\
\hline Filinia opolienis & $x$ & $x$ & $\mathrm{X}$ & Cyclopoida & $x$ & $x$ & $x$ \\
\hline Filinia saltator & $\mathrm{X}$ & $x$ & $\mathrm{x}$ & Copepodito & $x$ & $\mathrm{x}$ & $x$ \\
\hline Filinia sp. & $x$ & & & Copepodito ciclopoida & $\mathrm{X}$ & $\mathrm{X}$ & $x$ \\
\hline Filinia terminalis & $x$ & $x$ & $x$ & Harpacticoida & $x$ & $x$ & $x$ \\
\hline Flosculariidae & $x$ & & & Mesocyclops meridianus & $x$ & & \\
\hline Hexarthra intermedia brasiliensis & $x$ & & & Mesocyclops sp. & $x$ & & $x$ \\
\hline Hexarthra sp. & $x$ & $\mathrm{X}$ & $\mathrm{X}$ & Metacyclops sp. & $x$ & $x$ & $x$ \\
\hline Keratella amerciana & $x$ & $x$ & $x$ & Microcyclops sp. & $x$ & $\mathrm{X}$ & $x$ \\
\hline Keratella cochlearis & $x$ & $x$ & $x$ & Nauplio & $x$ & $\mathrm{X}$ & $x$ \\
\hline Keratella lenzi & $x$ & $\mathrm{X}$ & $x$ & Nauplio Calanoida & $x$ & $\mathrm{X}$ & $\mathrm{X}$ \\
\hline Keratella tropica & $x$ & $x$ & $x$ & Nauplio Cyclopoida & $x$ & $x$ & $\mathrm{x}$ \\
\hline Keratellla lenzi & & & $x$ & Notodiapotmus sp. & $x$ & $x$ & $x$ \\
\hline Lecane amazonica & $X$ & $X$ & $x$ & Notodiaptomus henseni & $x$ & & \\
\hline Lecane bulla & $x$ & $x$ & $x$ & Odontodiaptomus sp. & & $x$ & \\
\hline Lecane bulla bulla & $x$ & $x$ & $x$ & Paracyclops sp. & $\mathrm{X}$ & & \\
\hline Lecane closterocerca & & & $\mathrm{x}$ & Parastenocaris fontinalis & $\mathrm{X}$ & & \\
\hline Lecane cornuta & $x$ & & & Thermocyclops minutus & $x$ & $\mathrm{X}$ & $x$ \\
\hline Lecane cornuta & & & $x$ & Thermocyclops sp. & $x$ & $x$ & $x$ \\
\hline Lecane curvicornis & $X$ & & & Cladocerans & $\mathrm{BF}$ & DU & AF \\
\hline Lecane curvicornis curvicornis & $x$ & $\mathrm{X}$ & $x$ & Acroperus harpae & & & $x$ \\
\hline Lecane curvicornis nitida & & & $x$ & Acroperus sp. & & & $x$ \\
\hline Lecane elsa & & & $x$ & Alona guttata & $x$ & & \\
\hline Lecane haliclysta & $X$ & $x$ & & Alona sp. & $x$ & & $X$ \\
\hline Lecane hamata & & & $x$ & Alonella dadayi & $x$ & $x$ & $x$ \\
\hline Lecane leontina & $x$ & & $\mathrm{X}$ & Alonella sp. & $x$ & & \\
\hline Lecane limnetica & & & $x$ & Bosmina longirostris & $x$ & $x$ & $x$ \\
\hline Lecane ludwigii & $x$ & & $x$ & Bosmina cf. Longirostris & $x$ & & \\
\hline Lecane ludwigii $f$. Ohiensis & & $\mathrm{X}$ & & Bosmina sp. & $x$ & & \\
\hline Lecane ludwigii ludwigii & & $x$ & & Bosminopsis deiterrsi & $x$ & $x$ & $x$ \\
\hline Lecane luna & & & $\mathrm{X}$ & Camptocercus sp. & & & $x$ \\
\hline Lecane lunaris & $x$ & $x$ & $\mathrm{X}$ & Ceriodaphania cornuta & $x$ & $\mathrm{x}$ & $x$ \\
\hline Lecane lunaris crenata & & & $x$ & Ceriodaphnia quadrangula & $x$ & & \\
\hline Lecane monostyla & $x$ & $x$ & $x$ & Ceriodaphnia richardi & $x$ & & \\
\hline Lecane pyriformis & & $x$ & & Ceriodaphnia sp. & $x$ & $x$ & $x$ \\
\hline Lecane quadridentata & $\mathrm{X}$ & & $x$ & Chydorus eurynotus & & & $x$ \\
\hline Lecane signifera & & $x$ & $x$ & Chydorus parvireticulatus & $x$ & & \\
\hline Lecane sp. & $x$ & $x$ & & Chydorus sp. & $x$ & & $x$ \\
\hline Lecane stichaea & $x$ & & & Chydorus sphaericus & & & $x$ \\
\hline Lecane subtilis & $x$ & $x$ & & Daphnia gessneri & $x$ & $x$ & $x$ \\
\hline Lecane thienemanne & $x$ & & $x$ & Daphnia sp. & $x$ & $x$ & $x$ \\
\hline Lecane ungulata & & $x$ & $x$ & Diaphanosoma birgei & $x$ & & \\
\hline Lepadella benjamini & $X$ & $x$ & $x$ & Diaphanosoma brachyurum & $x$ & & \\
\hline Lepadella ovalis & & $x$ & $x$ & Diaphanosoma sp & $x$ & $x$ & $x$ \\
\hline Lepadella sp. & $x$ & & & Disparalona dadayi & $\mathrm{X}$ & & $x$ \\
\hline Lophocharis sp. & & & $x$ & Disparalona hamata & & & $x$ \\
\hline Macrochaetus collinsi & & & $x$ & Disparalona sp. & $x$ & & \\
\hline Macrochaetus sericus & & & $x$ & Ephemeroporus hybridus & $x$ & & $x$ \\
\hline
\end{tabular}


Table 1. Continued...

\begin{tabular}{|c|c|c|c|c|c|c|c|}
\hline Rotifers & BF & DU & AF & Rotifers (...continuing...) & BF & DU & AF \\
\hline Manfredium eudactylota euchla & $\mathrm{X}$ & & & Graptoleberis testudinaria & & & $\mathrm{X}$ \\
\hline Monommata sp. & $x$ & & & Ilyocriptus spinifer & $x$ & & $\mathrm{X}$ \\
\hline Mytilina macrocera & $x$ & & $\mathrm{X}$ & Ilyocryptus sp. & & & $\mathrm{X}$ \\
\hline Mytilina mucronata & & & $x$ & Kurzia latissima & $\mathrm{X}$ & & $\mathrm{x}$ \\
\hline Mytilina sp. & $\mathrm{X}$ & & & Leydigiopsis curvirostris & & & $\mathrm{X}$ \\
\hline Mytilina ventralis & $\mathrm{X}$ & & & Leydigiopsis sp. & $\mathrm{X}$ & $\mathrm{X}$ & $\mathrm{X}$ \\
\hline Platias leloupi f. Latiscapularis & $x$ & & & Macrothrix sp. & $x$ & & \\
\hline Plationus patulus macracanthus & $x$ & $\mathrm{X}$ & $\mathrm{X}$ & Macrothrix triserialis & $x$ & & $\mathrm{X}$ \\
\hline Plationus patulus patulus & $x$ & $\mathrm{x}$ & $\mathrm{X}$ & Moinodaphnia sp. & & $\mathrm{X}$ & \\
\hline Platyas quadricornis & $x$ & & & Notoalona sculpta & $X$ & & $\mathrm{X}$ \\
\hline Platyias cf. Leloupi & $x$ & $\mathrm{X}$ & $\mathrm{X}$ & Pseudochydorus globosus & $x$ & & \\
\hline Platyias quadricornis & $\mathrm{X}$ & $\mathrm{X}$ & $\mathrm{X}$ & Scapholeberis sp. & $\mathrm{X}$ & & \\
\hline Pleosoma lenticulare & & & $x$ & Simocephalus sp. & & & $\mathrm{X}$ \\
\hline
\end{tabular}

It should be read:

Table 1. Full list of zooplankton taxa (separated by rotifers, copepods and cladocerans) sampled before, during and after the formation of the reservoir lake from the hydropower dam UHE Colíder.

\begin{tabular}{|c|c|c|c|c|c|c|c|}
\hline Rotifers & BF & DU & AF & Rotifers (...continuing...) & BF & DU & AF \\
\hline Ascomorpha sp. & $\mathrm{X}$ & $\mathrm{X}$ & $\mathrm{X}$ & Polyarthra dolichoptera & $\mathrm{x}$ & & \\
\hline Asplanchna brightwellii & & & $x$ & Polyarthra remata & $x$ & & \\
\hline Asplanchna sieboldii & $\mathrm{X}$ & & & Polyarthra sp. & $x$ & $\mathrm{X}$ & $\mathrm{X}$ \\
\hline Asplanchna sp. & $x$ & $\mathrm{X}$ & $\mathrm{X}$ & Polyarthra vulgaris & $\mathrm{x}$ & & \\
\hline Bdelloidea & $x$ & $x$ & $x$ & Scaridium sp. & $\mathrm{x}$ & & $\mathrm{X}$ \\
\hline Beauchampiella sp. & $x$ & & $x$ & Synchaeta sp. & & $\mathrm{X}$ & $\mathrm{X}$ \\
\hline $\begin{array}{l}\text { Brachionus calyciflorus } f \text {. } \\
\text { amphicerus }\end{array}$ & $x$ & & & Testudinela ohlei & $\mathrm{X}$ & $x$ & $x$ \\
\hline Brachionus dolabratus & $\mathrm{X}$ & $\mathrm{X}$ & $x$ & Testudinella ahlstrom & $\mathrm{X}$ & & $\mathrm{X}$ \\
\hline Brachionus falcatus & & $x$ & $x$ & Testudinella emarginula & $x$ & & \\
\hline Brachionus mirus & & $x$ & $x$ & Testudinella mucronata & $x$ & & $\mathrm{X}$ \\
\hline Brachionus mirus angustus & $\mathrm{X}$ & & & Testudinella ohlei & $\mathrm{X}$ & & \\
\hline Brachionus mirus laticaudatus & $x$ & & & Testudinella patina & $\mathrm{x}$ & $\mathrm{X}$ & $\mathrm{X}$ \\
\hline Brachionus mirus mirus & $x$ & & & Testudinella sp. & $x$ & & $x$ \\
\hline $\begin{array}{l}\text { Brachionus quadridentatus } \\
\text { quadridentatus }\end{array}$ & & & $\mathrm{X}$ & Testudinella tridentata & $\mathrm{x}$ & & $\mathrm{X}$ \\
\hline Brachionus tropica & & $\mathrm{X}$ & & Trichocerca sp. & $\mathrm{X}$ & $\mathrm{X}$ & $\mathrm{X}$ \\
\hline Brachionus zahniseri & $\mathrm{X}$ & $\mathrm{X}$ & $x$ & Trichocerca mus & & & $\mathrm{X}$ \\
\hline Conochilus coenobasis & $x$ & & & Trichotia tetractis & $x$ & $x$ & $x$ \\
\hline Conochilus dossuarius & $x$ & & $\mathrm{X}$ & Copepods & $\mathrm{BF}$ & DU & AF \\
\hline Conochilus sp. & $x$ & $\mathrm{X}$ & $x$ & Argyrodiaptomus robertsonae & & & $x$ \\
\hline Dipleuchlanis propatula & $x$ & & $x$ & Attheyella sp. & $\mathrm{X}$ & & \\
\hline Filinia saltator & & & $x$ & Calanoida & $x$ & $x$ & $\mathrm{X}$ \\
\hline Filinia limnetica & $\mathrm{x}$ & $\mathrm{x}$ & $\mathrm{x}$ & Cyclopoida & $x$ & $x$ & $\mathrm{X}$ \\
\hline Filinia longiseta & $x$ & $\mathrm{x}$ & & Copepodid & $\mathrm{x}$ & $\mathrm{X}$ & $\mathrm{X}$ \\
\hline Filinia opolienis & $x$ & $\mathrm{x}$ & $\mathrm{X}$ & Copepodid ciclopoida & $\mathrm{x}$ & $\mathrm{X}$ & $\mathrm{X}$ \\
\hline Filinia saltator & $x$ & $x$ & $x$ & Harpacticoida & $\mathrm{x}$ & $x$ & $x$ \\
\hline Filinia sp. & $x$ & & & Mesocyclops meridianus & $\mathrm{X}$ & & \\
\hline Filinia terminalis & $x$ & $\mathrm{X}$ & $x$ & Mesocyclops sp. & $\mathrm{X}$ & & $\mathrm{X}$ \\
\hline Flosculariidae & $x$ & & & Metacyclops sp. & $x$ & $\mathrm{X}$ & $\mathrm{X}$ \\
\hline Hexarthra intermedia brasiliensis & $x$ & & & Microcyclops sp. & $x$ & $x$ & $x$ \\
\hline Hexarthra sp. & $x$ & $x$ & $x$ & Nauplii & $x$ & $x$ & $x$ \\
\hline Keratella amerciana & $x$ & $x$ & $x$ & Nauplii Calanoida & $x$ & $x$ & $x$ \\
\hline Keratella cochlearis & $X$ & $X$ & $x$ & Nauplii Cyclopoida & $X$ & $x$ & $X$ \\
\hline
\end{tabular}


Table 1. Continued...

\begin{tabular}{|c|c|c|c|c|c|c|c|}
\hline Rotifers & BF & DU & AF & Rotifers (...continuing...) & BF & DU & AF \\
\hline Keratella lenzi & $\mathrm{X}$ & $\mathrm{X}$ & $\mathrm{X}$ & Notodiaptomus sp. & $\mathrm{X}$ & $\mathrm{X}$ & $\mathrm{X}$ \\
\hline Keratella tropica & $x$ & $x$ & $x$ & Notodiaptomus henseni & $\mathrm{X}$ & & \\
\hline Lecane amazonica & $x$ & $x$ & $x$ & Odontodiaptomus sp. & & $x$ & \\
\hline Lecane bulla & $x$ & $x$ & $x$ & Paracyclops sp. & $x$ & & \\
\hline Lecane closterocerca & & & $x$ & Parastenocaris fontinalis & $x$ & & \\
\hline Lecane cornuta & $x$ & & $x$ & Thermocyclops minutus & $x$ & $x$ & $x$ \\
\hline Lecane curvicornis & $x$ & & & Thermocyclops sp. & $x$ & $x$ & $\mathrm{X}$ \\
\hline Lecane curvicornis curvicornis & $x$ & $\mathrm{X}$ & $\mathrm{X}$ & Cladocerans & $\mathrm{BF}$ & DU & $\mathrm{AF}$ \\
\hline Lecane curvicornis nitida & & & $x$ & Acroperus sp. & & & $x$ \\
\hline Lecane elsa & & & $x$ & Acroperus tupinamba & & & $x$ \\
\hline Lecane haliclysta & $x$ & $x$ & & Alona cf. guttata & $x$ & & \\
\hline Lecane hamata & & & $x$ & Alona sp. & $x$ & & $x$ \\
\hline Lecane leontina & $\mathrm{X}$ & & $x$ & Alonella dadayi & $x$ & $X$ & $x$ \\
\hline Lecane limnetica & & & $x$ & Alonella sp. & $x$ & & \\
\hline Lecane ludwigii & $x$ & & $x$ & Bosmina longirostris & $x$ & $X$ & $X$ \\
\hline Lecane ludwigii f. ohiensis & & $\mathrm{X}$ & & Bosmina cf. longirostris & $x$ & & \\
\hline Lecane ludwigii ludwigii & & $x$ & & Bosmina sp. & $\mathrm{X}$ & & \\
\hline Lecane luna & & & $x$ & Bosminopsis deiterrsi & $x$ & $x$ & $x$ \\
\hline Lecane lunaris & $x$ & $x$ & $x$ & Camptocercus sp. & & & $x$ \\
\hline Lecane lunaris crenata & & & $x$ & Ceriodaphania cornuta & $x$ & $x$ & $x$ \\
\hline Lecane monostyla & $\mathrm{X}$ & $x$ & $x$ & Ceriodaphnia quadrangula & $x$ & & \\
\hline Lecane pyriformis & & $x$ & & Ceriodaphnia richardi & $\mathrm{X}$ & & \\
\hline Lecane quadridentata & $x$ & & $x$ & Ceriodaphnia sp. & $x$ & $\mathrm{X}$ & $x$ \\
\hline Lecane signifera & & $x$ & $x$ & Chydorus eurynotus & & & $x$ \\
\hline Lecane sp. & $x$ & $x$ & & Chydorus parvireticulatus & $x$ & & \\
\hline Lecane stichaea & $x$ & & & Chydorus sp. & $x$ & & $x$ \\
\hline Lecane subtilis & $x$ & $x$ & & Chydorus sphaericus & & & $x$ \\
\hline Lecane thienemanne & $x$ & & $x$ & Daphnia gessneri & $\mathrm{X}$ & $\mathrm{X}$ & $x$ \\
\hline Lecane ungulata & & $\mathrm{X}$ & $x$ & Daphnia sp. & $x$ & $x$ & $x$ \\
\hline Lepadella benjamini & $x$ & $x$ & $x$ & Diaphanosoma birgei & $x$ & & \\
\hline Lepadella ovalis & & $x$ & $\mathrm{X}$ & Diaphanosoma brachyurum & $\mathrm{X}$ & & \\
\hline Lepadella sp. & $x$ & & & Diaphanosoma sp. & $x$ & $x$ & $x$ \\
\hline Lophocharis sp. & & & $x$ & Disparalona dadayi & $x$ & & $x$ \\
\hline Macrochaetus collinsi & & & $x$ & Disparalona hamata & & & $x$ \\
\hline Macrochaetus sericus & & & $x$ & Disparalona sp. & $x$ & & \\
\hline Manfredium eudactylota euchla & $x$ & & & Ephemeroporus hybridus & $\mathrm{X}$ & & $x$ \\
\hline Monommata sp. & $x$ & & & Graptoleberis testudinaria & & & $x$ \\
\hline Mytilina macrocera & $x$ & & $X$ & Ilyocryptus spinifer & $X$ & & $x$ \\
\hline Mytilina mucronata & & & $\mathrm{X}$ & Ilyocryptus sp. & & & $x$ \\
\hline Mytilina sp. & $x$ & & & Kurzia latissima & $x$ & & $x$ \\
\hline Mytilina ventralis & $x$ & & & Leydigiopsis curvirostris & & & $x$ \\
\hline Platias leloupi f. latiscapularis & $x$ & & & Leydigiopsis sp. & $x$ & $x$ & $x$ \\
\hline Plationus patulus macracanthus & $x$ & $\mathrm{X}$ & $X$ & Macrothrix sp. & $x$ & & \\
\hline Plationus patulus patulus & $x$ & $x$ & $\mathrm{X}$ & Macrothrix triserialis & $\mathrm{X}$ & & $x$ \\
\hline Platyas quadricornis & $x$ & & & Moinodaphnia sp. & & $x$ & \\
\hline Platyias cf. leloupi & $x$ & $x$ & $x$ & Notoalona sculpta & $\mathrm{X}$ & & $x$ \\
\hline Platyias quadricornis & $x$ & $x$ & $x$ & Pseudochydorus globosus & $x$ & & \\
\hline Ploesoma lenticulare & & & $x$ & Scapholeberis sp. & $x$ & & \\
\hline Ploesoma sp. & $x$ & & & Simocephalus sp. & & & $x$ \\
\hline Ploesoma truncatum & & & $\mathrm{X}$ & & & & \\
\hline
\end{tabular}


Were it reads:

Table 3. Typical taxon or taxa stage (when not possible to identify) for each period identified as significantly different from a null expectation in Indicator Value (IndVal) analysis. The Indicator value is shown for the significant species in each period: during $(\mathrm{DU})$ and after $(\mathrm{AF})$ the reservoir lake formation. There was no typical species identified before reservoir formation.

\begin{tabular}{llccc}
\hline \multicolumn{1}{c}{ Taxon or stage } & Period & IndVal & P \\
\hline Rotifers & Trichocerca sp. & DU & 0.763 & 0.047 \\
& Keratella coclearis & DU & 0.580 & 0.005 \\
Brachionus falcatus & DU & 0.574 & 0.034 \\
Asplanchna brightwellii & AF & 0.900 & 0.001 \\
Keratella americana & AF & 0.688 & 0.002 \\
Lecane amazonica & AF & 0.665 & 0.002 \\
Synchaeta sp. & AF & 0.597 & 0.003 \\
Lecane leontina & AF & 0.586 & 0.009 \\
Testudinella & AF & 0.549 & 0.006 \\
mucronata & & & \\
Lecane elsa & AF & 0.500 & 0.003 \\
Testudinella tridentata & AF & 0.496 & 0.008 \\
& Filinia opoliensis & AF & 0.470 & 0.046 \\
Odontodiaptomus sp. & DU & 0.400 & 0.027 \\
Copepodito & DU & 0.378 & 0.039 \\
& Cyclopoida & AF & 0.900 & 0.001 \\
& Mesocyclops sp. & AF & 0.589 & 0.002 \\
& Nauplio Cyclopoida & AF & 0.500 & 0.008 \\
Nauplio Calanoida & AF & 0.400 & 0.018 \\
\hline
\end{tabular}

It should be read:

Table 3. Typical taxon or taxa stage (when not possible to identify) for each period identified as significantly different from a null expectation in Indicator Value (IndVal) analysis. The Indicator Value is shown for the significant species in each period: before $(\mathrm{BF})$, during $(\mathrm{DU})$ and after $(\mathrm{AF})$ the reservoir lake formation.

\begin{tabular}{llccc}
\hline \multicolumn{1}{c}{ Taxon or stage } & Period & IndVal & P \\
\hline Rotifers & Trichocerca sp. & DU & 0.763 & 0.047 \\
& Keratella cochlearis & DU & 0.580 & 0.005 \\
Brachionus falcatus & DU & 0.574 & 0.034 \\
Asplanchna brightwellii & AF & 0.900 & 0.001 \\
Keratella americana & AF & 0.688 & 0.002 \\
& Lecane amazonica & AF & 0.665 & 0.002 \\
Synchaeta sp. & AF & 0.597 & 0.003 \\
& Lecane leontina & AF & 0.586 & 0.009 \\
Testudinella mucronata & AF & 0.549 & 0.006 \\
& Lecane elsa & AF & 0.500 & 0.003 \\
& Testudinella tridentata & AF & 0.496 & 0.008 \\
& Filinia opoliensis & AF & 0.470 & 0.046 \\
& Odontodiaptomus sp. & DU & 0.400 & 0.027 \\
& Copepodid & DU & 0.378 & 0.039 \\
& Cyclopoida & AF & 0.900 & 0.001 \\
& Mesocyclops sp. & AF & 0.589 & 0.002 \\
& Nauplii Cyclopoida & AF & 0.500 & 0.008 \\
& Nauplii Calanoida & AF & 0.400 & 0.018 \\
\hline
\end{tabular}

